\title{
Raman spectroscopy for diagnosis of calcification in human heart valves ${ }^{1}$
}

\author{
Enrique Uceda Otero ${ }^{\text {a }}$, Sokki Sathaiah ${ }^{\mathrm{a}, *}$, Landulfo Silveira Jr. ${ }^{\text {a }}$, \\ Pablo Maria Alberto Pomerantzeff ${ }^{\mathrm{b}}$ and Carlos Augusto Gonçalves Pasqualucci ${ }^{\mathrm{c}}$

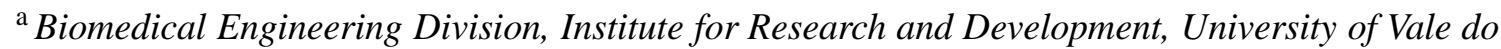 \\ Paraíba - UNIVAP, Av. Shishima Hifumi, 2911 Urbanova, ZIP: 12244-456, São José dos Campos, SP, \\ Brazil \\ ${ }^{\mathrm{b}}$ Institute of Heart, University of São Paulo - USP, Av. Dr. Enéas de Carvalho Aguiar, \\ 44 Cerqueira César, ZIP: 05403-000, São Paulo, SP, Brazil \\ ${ }^{\mathrm{c}}$ Department of Cardiovascular Pathology, Faculty of Medicine, University of São Paulo - USP, \\ Av. Dr. Arnaldo, 455, Cerqueira César, ZIP: 01246-903, São Paulo, SP, Brazil
}

\begin{abstract}
Near-Infrared Raman Spectroscopy (NIRS) has an excellent potential for a rapid, a less invasive and real time diagnosis of various human diseases. The objective of the present study was to apply NIRS for diagnosis of human heart valves and to develop a feasible algorithm to classify the valvular lesions. For Raman studies, a Ti:sapphire laser pumped by an argon laser provided $830 \mathrm{~nm}$ excitation. A spectrograph in conjunction with a liquid $\mathrm{N}_{2}$-cooled CCD detected Raman spectra. A total of 97 fragments of human heart valves were scanned and Raman results were compared with histopathology. Spectra were randomly separated into training and prospective groups. Raman data along with Principal Components Analysis (PCA) and Mahalanobis distance were used to model an algorithm for tissue classification, into two categories: normal (N), and calcified (C) heart valves. It has been found that, for $\mathrm{N}$ valves, the algorithm has sensitivities of $95 \%, 100 \%$ and specificities of $100 \%, 100 \%$ for training and prospective groups, respectively. For $\mathrm{C}$ valves the algorithm provided sensitivities of 100 and $100 \%$ and specificities of 95 and $100 \%$ for training and prospective groups, respectively. In conclusion, an algorithm has been developed and successfully applied for NIRS diagnosis of human heart valves with high sensitivities and specificities.
\end{abstract}

Keywords: Human heart valves, diagnosis, Raman spectroscopy, principal components analysis (PCA)

\section{Introduction}

The valvular cardiopathy disorder remains to be one of the major causes of deaths in the industrialized nations. Among the various causes of valves complications, the main cause of sudden death is due to aortic stenosis calcification, which occurs as a deposition of calcium minerals in the mitral valve annulus and the aortic valve. Calcification of the mitral valve could be indicative of valves dysfunction [1-3]. Calcification of the aortic valve is common in congenital as well as in acquired valve disease due to different etiologies [2].

The clinical complications associated with heart dysfunction are the leading causes of total deaths in the world. With the increasing number of people that survive the seventh decade and more it is important

\footnotetext{
${ }^{1}$ Paper presented at the First International Conference on Biomedical Spectroscopy: From Molecules to Men, Cardiff, 2002.

*Corresponding author: Sokki Sathaiah, Professor, Group of Diagnosis and Laser Therapy, Biomedical Engineering Division, Instituto de Pesquisa e Desenvolvimento - IP\&D, Universidade do Vale do Paraíba - UNIVAP, Av. Shishima Hifumi, Urbanova, 2911, ZIP: 12244-000, São José dos Campos, SP, Brazil. Tel.: +55 123947 1124; Fax: +55 1239471149 ; E-mail: sokki@univap.br.
} 
to know the alterations in the cardiovascular system that occur frequently with the aging. Normally, aging causes alterations at the pericardia, cardiac chambers, coronary arteries, epicardycs, neural conduction system, sides of aorta as well as the miocardy and the iris of cardiac valves [3].

The molecular mechanisms of calcification of bicuspid, rheumatic or degenerative valves are unclear [4]. Various depositions with tissue compositions and morphologies similar to those in cartilage, organized lamellar bone, osteoclast, osteoblast, as well as lymphocytes and macrophages have been found in the calcified valves.

Optical spectroscopy techniques, such as reflectance, fluorescence, infrared absorption and Raman scattering can provide information about the tissue composition at the molecular level. Among these techniques, Raman spectroscopy has an excellent capability to provide valuable biochemical information for nondestructive diagnosis of cardiovascular disorders [5-10].

Biological samples are often living systems that undergo complex reactions or interactions. Thus, the knowledge of the molecular dynamics of the systems is crucial to understand the mechanism of diseases. Raman spectroscopy and its variations (such as Dispersive/FT Raman, Raman microscopy and resonance Raman spectroscopies), have been emerging as suitable methods for better understanding of the molecular basis of the various human diseases [5-15].

Raman effect is a fundamental process in which energy is exchanged between light and matter. The process of Raman scattering can be viewed as the transition of a molecule from its ground state to an excited vibrational state, accompanied by the simultaneous absorption of a incident photon and emission of a Raman scattered photon [16].

The Raman spectrum of a particular tissue exhibits narrow, well resolved bands revealing the presence of many biochemicals. The relative contributions of these biochemicals to tissue Raman spectrum are proportional to their relative concentrations in the tissue. The quantitative nature of Raman spectra, combined with the ability to provide unique fingerprints of the biochemicals presented in tissue, illustrate the potential of Raman spectroscopy for quantitative analysis [5-15].

Among various biomedical investigations of Raman spectroscopy, some of the important diagnosis applications are in the atherosclerosis [5-10], breast cancer [5,12], colon cancer [5,13], skin cancer [5] and blood samples [5,14,15].

In this work near-infrared Raman spectroscopy (NIRS) technique has been used to diagnose the calcification in human heart valves. The tissue Raman spectra are compared with histopathology (gold standard). An algorithm based on principal components analysis (PCA) combined with Mahalonobis distance (discriminant analysis) has been developed for tissue classification into two categories: normal $(\mathrm{N})$ and calcified $(\mathrm{C})$ heart valves.

\section{Materials and methods}

\subsection{Raman spectroscopy}

The details of the near-infrared Raman spectrometer used in this study are as described elsewhere [9]. Briefly, an argon laser (Spectra Physics, model stabilite 2017) pumps a Ti:sapphire laser (Spectra Physics, model 3900S) providing $150 \mathrm{~mW}$ of $\mathrm{CW}$ infrared light at $830 \mathrm{~nm}$. A bandpass filter (Kaiser Optical systems, model HLBF 830) at $830 \mathrm{~nm}$ filters the laser light. Steering optics are used to reflect and direct the laser beam to reach the sample holder. The sample holder is placed in front of the spectrograph with the laser beam reaching its central region with approximately $90^{\circ}$ excitation-collection geometry. 
A telescope with the numerical aperture matching with that of the spectrograph is used for scattered light collection. Notch rejection filter (Kaiser Optical Systems, model HSPF 830AR20) at $830 \mathrm{~nm}$ is used to block laser light from entering the spectrograph.

The spectrograph (Chromex, model 250IS) shows a spectral resolution of about $8 \mathrm{~cm}^{-1}$. After dispersion, the Raman signal is detected by a liquid-nitrogen cooled deep-depletion CCD with $1024 \times 256$ pixels (Princeton Instruments, model LN/CCD - 1024 - EHR1) in conjunction with a controller (Princeton Instruments, model ST130). The controller is connected to a personal computer (IBM, model Aptiva $\mathrm{K}-45-100 \mathrm{MHz}$ ). Winview ${ }^{\circledR}$ software (Princeton Instruments) is used to control the CCD readout, exposure time and data storage for post-processing. The exposure time for collecting Raman signal is $100 \mathrm{~s}$ in all experiments.

\subsection{Human heart valve samples}

Fragments of heart valves were obtained from the Autopsy Service of Medical School of University of São Paulo (São Paulo, SP, Brazil) taken from randomized cadavers. A total of 97 heart valves segments, approximately $5 \mathrm{~mm}$ long taken from 12 subjects, were extracted, snap-frozen and stored in liquid nitrogen $\left(-196^{\circ} \mathrm{C}\right)$. Prior to the Raman measurements, the samples were warmed up to room temperature and added them a few drops of $(0.9 \%)$ saline solution during Raman data acquisition.

\subsection{Histopathological analysis}

After the spectroscopy, the scanned spots where the laser incident on the valvular fragments were marked with India ink and the samples were immersed in $10 \%$ formalin solution, bottled and labeled. Traditional histopathological technique was performed. The histopathological classification was made by a board-certified pathologist to identify the presence or absence of lesions, aiming to correlate the Raman spectra to tissue type.

\subsection{Spectrum calibration and pre-processing}

All spectra were recorded in graphic format and converted to ASCII format for post-processing. The Raman wavenumber shift calibration was done using indene bands. The low frequency fluorescence background was removed by fitting and subtracting a 4th order polynomial from the gross spectrum. In order to prepare the data to perform PCA, all spectra were normalized to obtain scale-free intensity data. From the total of 97 spectra a group of 50 randomly selected spectra has been treated as a training data set and the remaining 47 spectra were used to test the Diagnostic Algorithm prospectively.

\subsection{Principal components analysis $(P C A)$}

PCA is a very effective data reduction technique. It decomposes the spectra into the most important spectral features (the principal components) based on the spectral variance. A few components are able to explain the most important differences observed. With the use of PCA a minimisation of the number of variables is obtained, which constitutes an orthogonal system forming a spectral basis, allowing to generate all the spectral space [17].

The PCs can be interpreted as a mathematical representation of main spectral features (eigenvectors). These eigenvectors are similar to Raman spectra with the presence of positive as well as negative peaks at the Raman spectral positions of biochemical components present in the cardiac valves. The scores are basically the intensities of each PC to reconstruct the original spectrum. 


\section{Results}

\subsection{Histopathological analysis}

Among the 97 fragments of cardiac valves considered in the study, 80 were classified as normal and 17 presented different stages of calcification and were classified as calcified.

For implementation of diagnostic algorithm based on PCA technique, spectra of the 97 fragments were randomly split into two groups, the training group with 50 spectra and the second, the prospective (test) group with 47 spectra. Considering the 50 fragments of cardiac valves of the training group, 41 were classified as normal and 9 as calcified valvular tissues on the basis of histopathological analysis. Whereas the 47 fragments from the group of prospective analysis, 39 were found to be normal and 8 to be as calcified valvular tissues (Table 1).

\subsection{Raman spectral analysis}

Typical Raman spectra of human cardiac valves are shown in Fig. 1. The main bands appearing in the spectrum of a normal valve (Fig. 1(a)) are primarily ascribed to structural proteins (collagen and elastin). The prevailing bands at 1660,1452 and $1260 \mathrm{~cm}^{-1}$ are ascribed to amide I (C=O stretching), $\mathrm{C}-\mathrm{H}$ bending $(\mathrm{H}-\mathrm{C}-\mathrm{H}$ union) and amide III vibrational modes of structural proteins, respectively $[5,7]$.

Raman spectrum of valvular tissue with calcification exhibits different characteristics compared to a normal valvular tissue spectrum. The observed major bands at 962 and $1073 \mathrm{~cm}^{-1}$ are ascribed to phosphate and carbonate symmetric stretching vibrations of accumulated calcium hydroxyapatite and of carbonate apatites (Fig. 1(b)). The presence of relatively intense bands in the region between 1200 to $1800 \mathrm{~cm}^{-1}$, particularly at 1260,1452 and $1660 \mathrm{~cm}^{-1}$ are presumably due to the presence of normal tissue components (proteins) that are embedded in the calcified valvular tissue $[5,7]$.

\section{Table 1}

A total of 97 human cardiac valvular samples, grouped into: training and prospective (to develop the diagnosis algorithm) based on histopathological results

\begin{tabular}{lcc}
\hline & Training group & Prospective group \\
\hline Normal cardiac valves & 41 & 39 \\
Calcified cardiac valves & 9 & 8 \\
Total samples & 50 & 47 \\
\hline
\end{tabular}

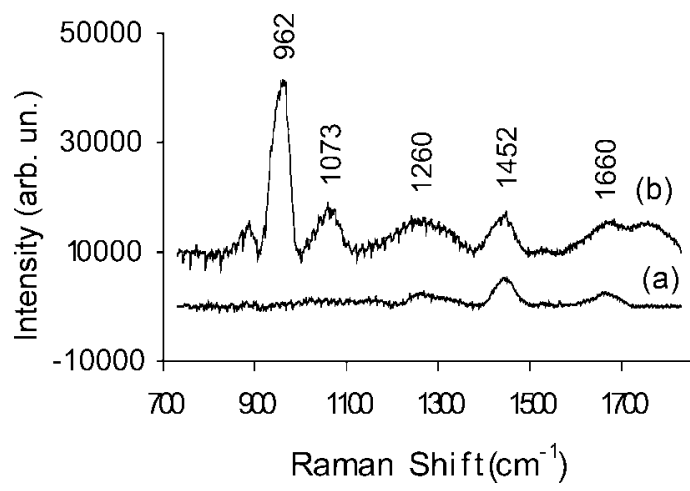

Fig. 1. Raman spectra (in vitro) of: (a) normal and (b) calcified lesions of human cardiac valves. 


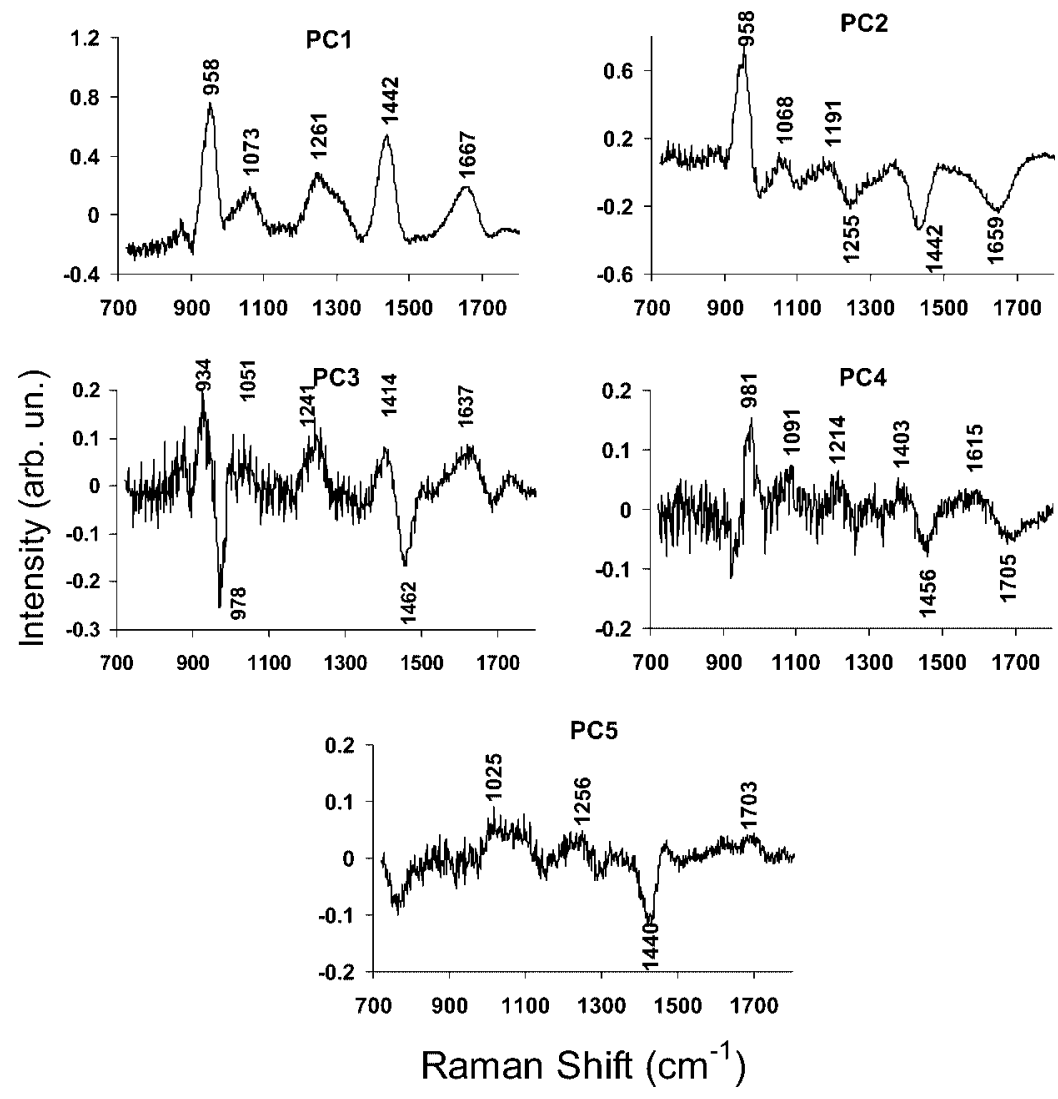

Fig. 2. First five principal components of Raman spectral training group. The spectral features in each PC are related to Raman spectral bands of biochemicals present in human cardiac valves.

\subsection{Principal components analysis}

The five most important PC vectors calculated from the training spectral group are shown in the Fig. 2 . The choice of which PCs could be used for diagnosis was based on the presence of main spectral bands that appear at positions related to the type of tissue under study [18].

In the proposed study, using the 50 spectra of the training group, the PCA algorithm determined that the PC1 to PC5 are the most important ones and are responsible for nearly $90 \%$ of all spectral variations.

Among the five PCs, the first (PC1) and second (PC2) principal components exhibit the majority of spectral characteristics related to a combination of normal and calcified valvular tissues $(958,1073,1261$, 1442 and $1667 \mathrm{~cm}^{-1}$ ). The PC3, PC4 and PC5 exhibit bands in positions with little importance for tissue classification and in some cases are very weak and appear and as a first derivative of original bands (the case of PC3, 934 and $978 \mathrm{~cm}^{-1}$ bands).

\subsection{Diagnostic algorithm based on PCs and Mahalanobis distance}

For implementation of diagnostic algorithm, the pair of PCs containing the most important spectral signatures to promote the split of the two categories was used. For that, the criterion of presence of important bands and the greater Mahalanobis distance of the scores from one category, for example, $\mathrm{N}$, in relation to scores from the remaining category, $\mathrm{C}$, has been worked out for all the five PCs. 
As can be seen from the Table 2, the greater Mahalanobis distances, normalized for each pair of PC scores, were obtained using the first and second PCs from training spectra (PC1 X PC2). The greater the Mahalanobis distance, greater is the separation between categories and lesser is the covariance (or dispersion) of data group. This result shows that greater Mahalanobis distance is obtained with the first and second scores is in accordance with the spectral characteristics information contained in each one of PCs, in which PC1 represent bands associated with a combination of calcified lesions and valvular walls without calcification, while PC2 represents bands related to calcified valvular walls.

Figure 3 shows a scattered plot of PC1 versus PC2 scores for Raman spectra of the training group. As it can be seen in the plot, there is a good separation of points in two categories, normal valvular tissue $(\mathrm{N})$ and calcified valvular tissue (C). To implement the algorithm for having clearly separated histopathological categories, separation surfaces were plot based on Mahalanobis distance. As mentioned above, the surfaces represent the average Mahalanobis distance between the PC scores of $\mathrm{N}$ and $\mathrm{C}$ valves.

With the aim to test the diagnostic algorithm prospectively, the five PC eigenvectors calculated from the training group (Fig. 2) were used to calculate what is the amount (score) of each PC that all the prospective spectra have. For that, a fitting routine based on least square minimization was made. Then, the PC1 and PC 2 scores, calculated by projecting each PC on each one of 47 prospective spectra, were also presented in a scattered plot, maintaining the same Mahalanobis surfaces obtained for the training group (Fig. 4).

Analysis of Fig. 3 shows that the diagnostic algorithm based on PCA applied to training group allowed that 39 out of 41 normal cardiac valve spectra were presented in concordance with histopathology, and 9 out of 9 calcified cardiac valvular spectra were correctly classified as such, but two normal valves were classified as calcified ones. In Fig. 4, the result of the diagnostic algorithm indicates that 39 out of 39

\section{Table 2}

Normalized Mahalanobis distance for pairs of scores from the five PCs compared to each combination of tissue histopathological categories using the training group

\begin{tabular}{lcccc}
\hline & PC2 X PC1 & PC2 X PC3 & PC2 X PC4 & PC2 X PC5 \\
\hline N/C & 0.664 & 0.732 & 0.454 & 1.000 \\
C/N & 1.000 & 0.040 & 0.042 & 0.039 \\
\hline
\end{tabular}

Notes: $\mathrm{N}$ - normal cardiac valve tissue; $\mathrm{C}$ - calcified cardiac valve tissue; $\mathrm{PC}$ - principal component score.

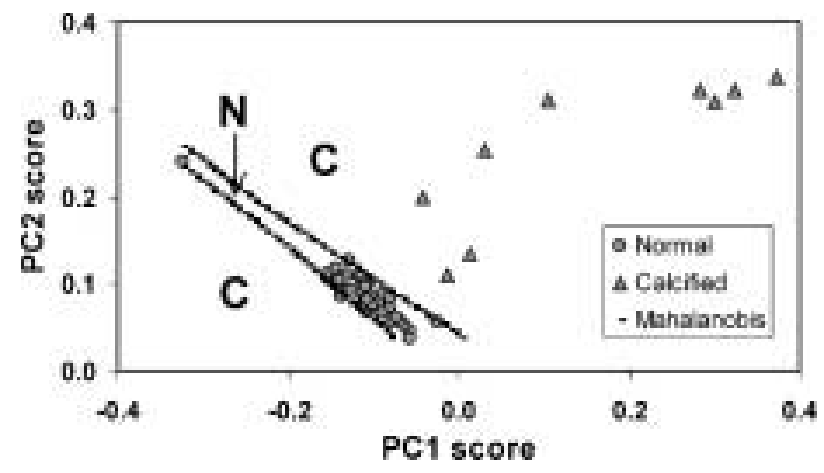

Fig. 3. Scatter plot of PC1 versus PC2 scores for the 50 Raman spectra used in the training group of the algorithm. Diagnosis surfaces (lines) separate spectrally similar categories. C - calcified region, $\mathrm{N}$ - normal region. 


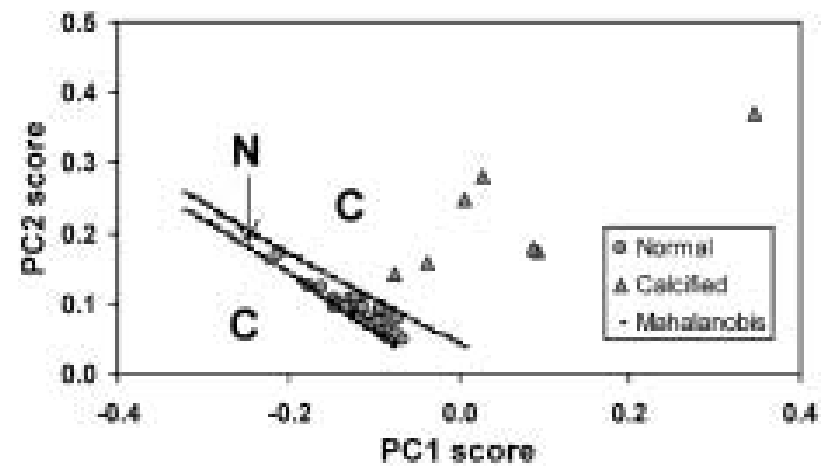

Fig. 4. Scatter plot of PC1 versus PC2 scores for the 47 Raman spectra of the prospective group using the same diagnostic surfaces calculated for the training group. $\mathrm{C}$ - calcified region, $\mathrm{N}$ - normal region.

Table 3

Comparison of results from the diagnostic algorithm, applied retrospectively to the training group, with the histopathological analysis

\begin{tabular}{lcc}
\hline & Histopathological diagnosis & PCA diagnosis \\
\hline Normal cardiac valves & 41 & 39 \\
Calcified cardiac valves & 9 & 11 \\
Total samples & 50 & 50 \\
\hline
\end{tabular}

Table 4

Comparison of results from the diagnostic algorithm, applied to the prospective group, with the histopathological analysis

\begin{tabular}{lcc}
\hline & Histopathological diagnosis & PCA diagnosis \\
\hline Normal cardiac valves & 39 & 39 \\
Calcified cardiac valves & 8 & 8 \\
Total samples & 47 & 47 \\
\hline
\end{tabular}

normal cardiac valvular spectra are presented in concordance with histopathology and 8 out of 8 calcified cardiac valves presented also correct classification. The results are summarized in Tables 3 and 4 .

\subsection{Sensitivity and specificity}

Sensitivity and specificity rates of the diagnosis algorithm based on PCA for each tissue category, for both training and prospective groups, are shown in Table 5. For normal cardiac valvular tissue diagnosis, it was found that algorithm had a high sensitivity of $95 \%$ and $100 \%$ for training and prospective groups, respectively, and a high enough specificity of $100 \%$ and $100 \%$ for both groups. For calcified cardiac valvular tissue, it was found that algorithm has a high enough sensitivity of $100 \%$ and $100 \%$ for both the groups, and a high specificity of $95 \%$ and $100 \%$ for training and prospective groups, respectively.

\section{Discussion and conclusion}

The use of Raman spectroscopy as diagnosis tool for pathological alterations is based on the fact that each biochemical component present in tissues has a characteristic vibrational spectrum, with sharp and 
Table 5

Sensitivity and specificity test results obtained with the diagnostic algorithm for the training and prospective groups

\begin{tabular}{lcc}
\hline & Sensitivity (\%) & Specificity (\%) \\
\hline Training group & & \\
$\quad$ Normal cardiac valves & 95 & 100 \\
Calcified cardiac valves & 100 & 95 \\
Prospective group & & \\
$\quad$ Normal cardiac valves & 100 & 100 \\
Calcified cardiac valves & 100 & 100 \\
\hline
\end{tabular}

well resolved bands, which is a digital imprint of biomolecules. The characterisation of valvular walls through Raman spectroscopy was done basically by the analysis of the differences of molecular composition in two different stages: (a) normal and (b) pathological or with calcification. In the spectrum of normal cardiac valve (Fig. 1), bands related to structural proteins, mainly due to collagen and elastin (which are the major constituents of normal valve) were observed. In cardiac valve with calcification, the predominant bands in the spectrum were due to calcium hydroxyapatite accumulation. The relatively less intense band at $1452 \mathrm{~cm}^{-1}$ reveals the presence of fibrous components that are mixed with the calcified tissue. The Raman spectrum, besides furnishing a qualitative diagnosis, it can be used to quantify collagen, elastin and hydroxyapatite deposits in valves.

The multivariate technique such as PCA presents an advantage that it needs a little or no knowledge of tissue constitution and usually a few PC eigenvectors are important among many spectra from different samples [18]. This facilitates the development, processing and validation of different classification algorithms.

The diagnostic algorithm based on PCA with the PC1 and PC2 scores for normal and calcified cardiac valvular diagnosis presented sensitivity and specificity for both training group and prospective group higher than $95 \%$.

In the implemented PCA algorithm, two samples with histopathological results of normal cardiac valves have been classified as calcified. Generally, biological tissues are very heterogeneous. Small differences in the band positions could bring to false diagnosis. It was observed that they are very close to the decision lines, showing that a small spectral difference is present. A lot of attention has been made to mark (with Indian ink) the points studied by Raman spectroscopy and to analyze the same points by the pathologist. The observed false positive cases could be due to errors related to marking or analyzing the marked points.

In this study, the algorithm presented results with high sensitivity and specificity. Further, Mahalanobis distance can be used as a discrimination criterion of histopathological categories. It presents advantages with respect to the empirical choice of decision lines since it takes into consideration the distance between the points to the center of the distribution as well as the covariance of the distribution.

These results suggest that near-infrared Raman spectroscopy can become a promising technique in therapeutic actions, such as laser angioplasty, being able to feedback the system that would identify the calcification in cardiac valves. The biochemical informations obtained through Raman spectroscopy can also be an assistant in real time diagnosis and in controlled treatment of human cardiac valve calcification. Understanding the mechanisms of cardiac valvular calcification could be important in prevention of atherosclerosis [4].

Finally, with the implementation of a dedicated Raman diagnostic system with DSPs (Digital Signal Processors) and a rapid data analysis algorithms [9,18-20], Raman fiber probes specially assembled for 
low fluorescence background [5,6,10,21], a compact spectrograph and a semiconductor laser, in vivo experiments could be done in near future.

\section{Acknowledgements}

Authors are grateful to PADCT/FINEP (Scientific and Technological Development Support Program - Brazil) through grant no. 5696015300, CNPq (National Council of Research - Brazil) through grant no. 300460/97, FAPESP (Research Support Foundation of São Pulo State - Brazil) through grant no. 02/05920-6 for the financial support. One of our authors (S.S.) would like to thank CNPq for the grant of "Individual: Research Productivity" award (Grant No. 300460/97; 303293/2003-9; 1997-2006).

\section{References}

[1] R. Schlant, Peri-operative evaluation and management of patients with known or cardiovascular disease who undergo noncardiac surgery, in: The Heart, R. Schlant and W. Alexander, eds, McGraw-Hill, New York, 1994, pp. 2421-2430.

[2] R.W. Wissler, Principles of the pathogenesis of atherosclerosis, in: Heart Disease, E. Braunwald, ed., W.B. Saunders, Philadelphia, 1980, pp. 1221-1245.

[3] M. Berne, and M. Levy, Cardiovascular Physiology, Mosby, New York, 1997.

[4] E. Molher, Molecular mechanics of aortic calcification, in: 48th Annual Scientific Session of American College of Cardiology, March 7-10, 1999, New Orleans.

[5] E.B. Hanlon, R. Manoharan, T.-W. Koo, K.E. Shafer, J.T. Motz, M. Fitzmaurice, J.R. Kramer, I. Itzkan, R.R. Dasari and M.S. Feld, Prospect for in vivo Raman spectroscopy, Physics in Medicine and Biology 45 (2000), R1-R59.

[6] S.W.E. Van de Poll, T.J. Römer, G.J. Puppels and A. Van der Laarse, Raman spectroscopy of atherosclerosis, Journal of Cardiovascular Risk 9 (2002), 255-261.

[7] R.P. Rava, J.J. Baraga and M.S. Feld, Near-infrared Fourier-transform Raman spectroscopy of human artery, Spectrochimica Acta A 47 (1991), 509-512.

[8] S. Sathaiah, L. Silveira Jr., C.A. Pasqualucci, R.A. Zangaro, M. C. Chavantes and M.T.T. Pacheco, Diagnosis of human coronary artery with near infrared Raman spectroscopy, in: XVth Int. Conf. on Raman Spectroscopy, S.A. Asher and P.B. Stein, eds, John Wiley \& Sons, New York, 1996, pp. 1120-1121.

[9] L. Silveira Jr., S. Sathaiah, R. A. Zângaro, M.T.T. Pacheco, M.C. Chavantes and C.A.G. Pasqualucci, Correlation between near-infrared Raman spectroscopy and the histopathological analysis of atherosclerosis in human coronary arteries, Lasers in Surgery and Medicine 30 (2002), 290-297.

[10] H.P. Buschman, E.T. Marpel, M.L. Wach, B. Benney, T.C.B. Schut, H.A. Bruining, A.V. Bruschke, L.A. Vander and G.J. Puppels, In vivo determination of the molecular composition of artery wall by intra-vascular Raman spectroscopy, Analytical Chemistry 72 (2000), 3771-3775.

[11] P.V. Huong, Raman spectroscopy for biological applications, in: Analytical Raman Spectroscopy, J.G. Grasselli and B.J. Bulkin, eds, John Wiley \& Sons, New York, 1991, pp. 397-424.

[12] R. Manoharan, K. Shafer, L. Perelman, J. Wu, K. Chen, G. Deinum, M. Fitzmaurice, J. Myles, J. Crowe, R.R. Dassari and M.S. Feld, Raman spectroscopy and fluorescence photon migration for breast cancer diagnosis and imaging, Photochemistry and Photobiology 67 (1998), 15-22.

[13] N.N. Boustany, R. Manoharan, R.R. Dasari and M.S. Feld, Ultraviolet resonance Raman spectroscopy of bulk and microscopic human colon tissue, Applied Spectroscopy 54 (2000), 24-30.

[14] Y. Jianan, C. Brian and D. Suria, Concentration measurements of multiple analytes in human sera by near-infrared laser Raman spectroscopy, Applied Optics 38 (1999), 5491-5497.

[15] A. Berger, T.W. Koo, I. Itzhan, G. Horowitz and M.S. Feld, Multi component blood analysis by near-infrared Raman spectroscopy, Applied Optics 38 (1999), 2916-2926.

[16] G. Turrel, The Raman effect, in: Raman Microscopy Developments and Applications, G. Turrel and J. Gorset, eds, Academic Press, London, 1996, pp. 1-25.

[17] C. Chatfield and A.J. Collins, Introduction to Multivariate Statistics, Chapman \& Hall, London, 1980, p. 246.

[18] G. Deinum, D. Rodriguez, T.J. Römer, M. Fitzmaurice, J.R. Kramer and M.S. Feld, Histological classification of Raman spectra of human coronary artery atherosclerosis using principal component analysis, Applied Spectroscopy 53 (1999), 938-942. 
[19] S. Hassan, A.R. Paula Jr. and S. Sathaiah, Real time signal processing system: application to biotechnological signals classification, in: II Conference on Management and Control of Production and Logistics; Session: Automatic Control and Factory Automation; Chapter A4, Copyright: International Federation of Automatic Control - IFAC, 2000, P340-1-P340-5.

[20] A.R. Paula Jr. and Sokki Sathaiah, Raman spectral classification of atherosclerosis using neural networks and discriminant analysis, in: Proc. Fourth IEEE's International Caracas Conference on Devices Circuits and Systems, ICCDCS Publications, Aruba, 2002, pp. 1021: 1-6.

[21] C.J. Lima, S. Sathaiah, L. Silveira Jr., R.A. Zângaro and M.T.T. Pacheco, Development of catheters with low fiber background signals for Raman spectroscopic diagnosis applications, Artificial Organs 24 (2000), 231-234. 


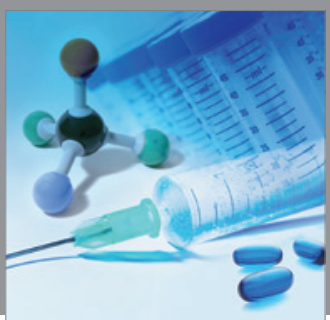

International Journal of

Medicinal Chemistry

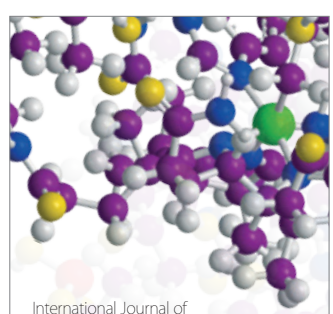

Carbohydrate Chemistry

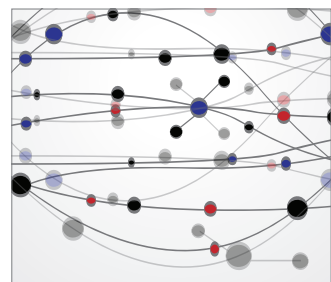

The Scientific World Journal
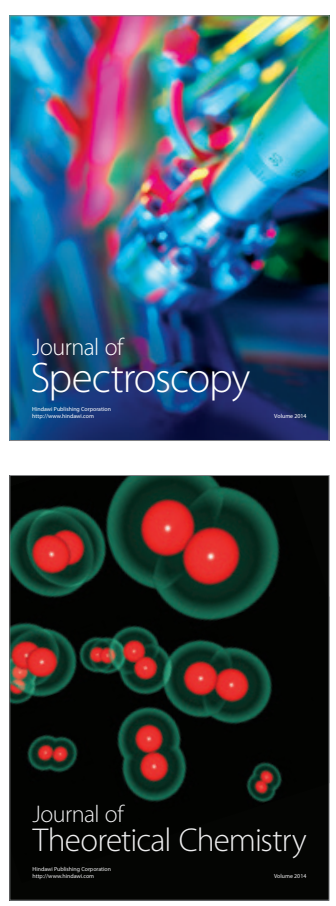
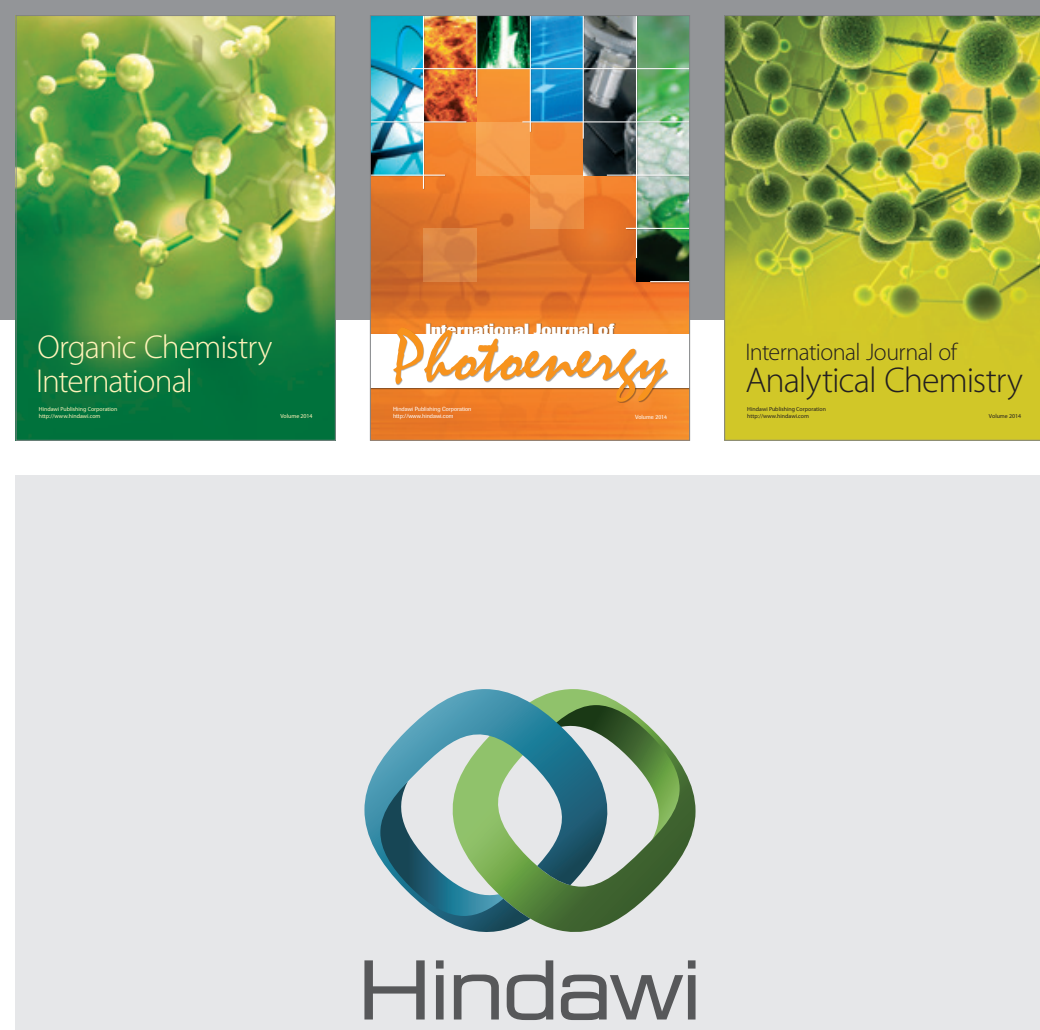

Submit your manuscripts at

http://www.hindawi.com
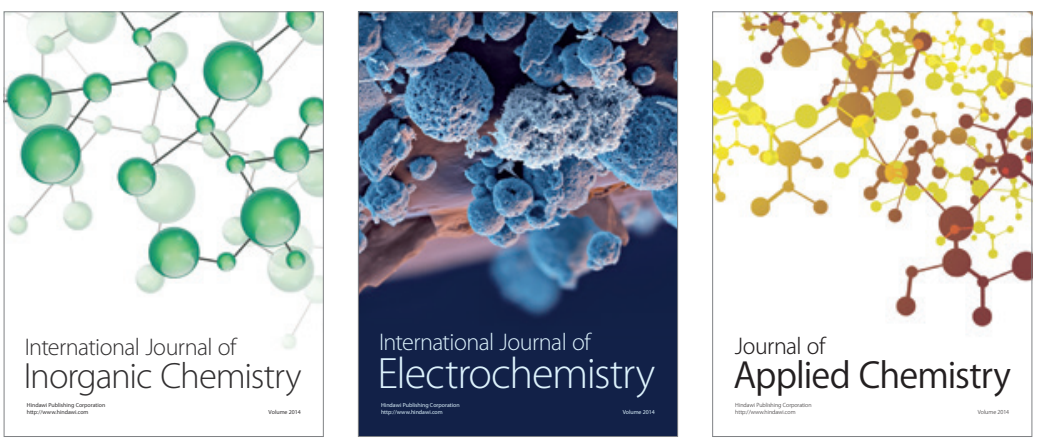

Journal of

Applied Chemistry
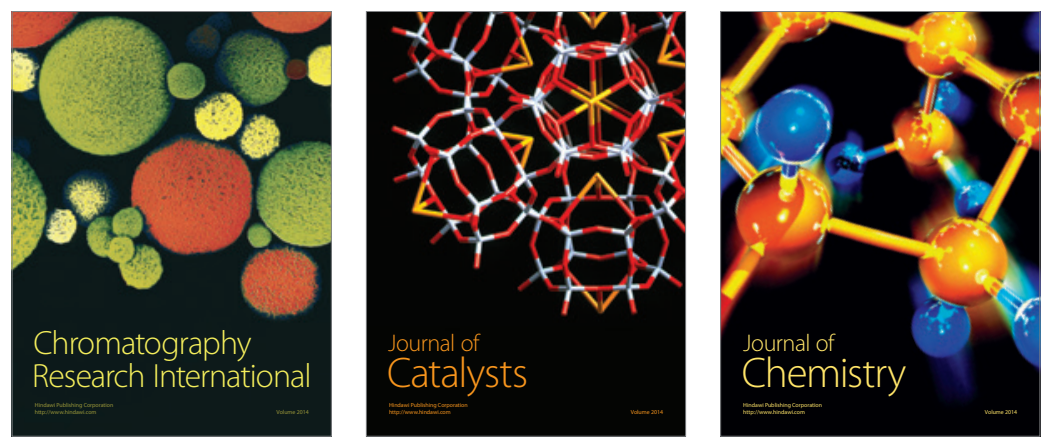
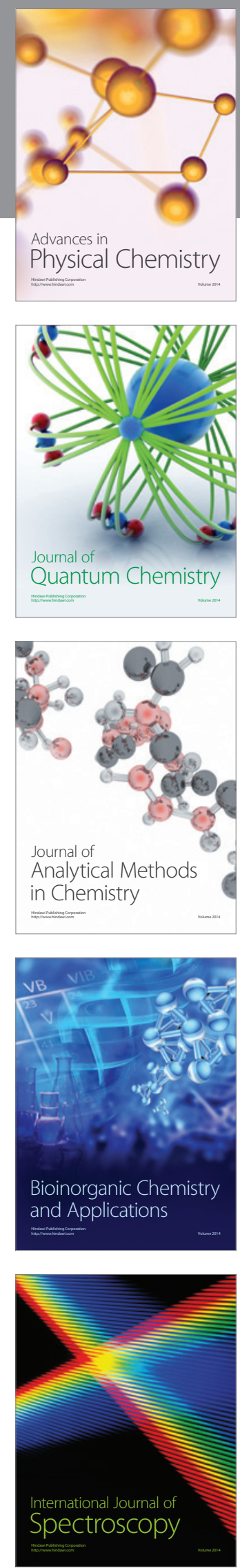